\title{
Industry 4.0 - Digital Twin Applied to Direct Digital Manufacturing
}

\author{
João Vitorino ${ }^{1, a^{*}}$, Eliseu Ribeiro ${ }^{2,3, b}$, Rúben Silva ${ }^{1, c}$, Cyril Santos $^{1, d}$, \\ Pedro Carreira ${ }^{1, e}$, Geoffrey Mitchell ${ }^{1, f}$ and Artur Mateus ${ }^{1, g}$ \\ ${ }^{1}$ CDRsp - Centre for rapid and sustainable product development, Rua de Portugal - Zona \\ Industrial, 2430-028 Marinha Grande, Portugal
${ }^{2}$ Escola Superior de Tecnologia e Gestão do Instituto Politécnico de Leiria, Campus 2 - Morro do Lena, Alto do Vieiro, Apt 4163, Edifício D, 2411-901 Leiria, Portugal
${ }^{3}$ INESCC - Instituto de Engenharia de Sistemas e Computadores de Coimbra, Pólo II, Rua Sílvio Lima, 3030-290 Coimbra, Portugal \\ ajoao.vitorino@ipleiria.pt, beliseu.ribeiro@ipleiria.pt, cruben.j.silva@ipleiria.pt,

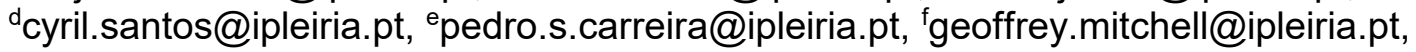 \\ gartur.mateus@ipleiria.pt
}

Keywords: Industry 4.0, Direct Digital Manufacturing, Digital Twin, Robot.

\begin{abstract}
Marinha Grande is a city in Leiria's district, Portugal. Marinha Grande is known as the moulding city, influenced by the glass, plastic and rapid manufacturing industry. Its history comes from the $18^{\text {th }}$ century with the first glass factory. In order to improve technological development in the local industry, Centre for rapid and sustainable product development (CDRsp) was established in 2007.

With that historical know-how and data-based moulding manufacturing, this work goal is to link that data with today's technology, implementing the Industry 4.0. That information would be stored in a Cloud-Based Design and Manufacturing (CBDM) as well as the real-time operational data. Accessing to that cloud, the design and production engineers can work together to digitally create a product without having to stop the machinery.

To implement these concepts, this paper suggests a Digital Twin (DT) to take advantage of the historical information allied to the existent industrial machinery. It suggests a digital twin of a robotic arm with an additive or hybrid manufacturing tool, printing big parts (e.g. garden benches or urban furniture) with reused materials such as tire, cork, wood or stone pow loads.
\end{abstract}

\section{State of the Art}

The Industry 4.0 concept was suggested, in 2011, by German's government to improve its economy [1], but quickly the concept is changed to a global scale. The concept is simple: link and gives "intelligence" to industrial machinery, improving operational efficiency and productivity [2], working together and give them independence to recognize what kind of task it is to do and to decide a solution to implement when a fault occurs. Industry 4.0 encompasses numerous technologies and associated paradigms such as Radio Frequency Identification (RFID), Enterprise Resource Planning (ERP), Internet of Things (IoT), cloud-based manufacturing, and social product development [3]. Same authors refer five features that Industry 4.0 add to industry: digitization, optimization, and customization of production; automation and adaptation; Human Machine Interaction (HMI); value-added services and businesses, and automatic data exchange; and communication $[1,3,4]$. Summarizing, Industry 4.0 is an industrial process of value adding and knowledge management [4].

The IoT is one of the most significant features and a mark of Industry 4.0. The RFID keeps all the information related to building a product in each product besides the IoT keep that information in a cloud to build the products. Now, IoT includes all type of devices ranging industrial machinery, automation systems, home appliances, watches, cars, etc. IoT can be described as a data exchange and internet-based communication between embedded systems of electrical, mechanical, 
computational and communication mechanisms. It is important that which device communicates with others, so arise the term Operational Technology (OT) referring to the hardware and software that allows the internet-based communication (e.g. Programmable Logic Controllers (PLC)). In industrial environments, OT could be called Industrial Control Systems (ICS). The implementation of ICS is currently happening in large scale across numerous industries, and it provides a technological alignment with the needs of future smart manufacturing systems and Industry 4.0. [2]

Some communication protocols had been developed to able communication between "things". Nowadays, two protocols are used in large scale: OPC UA (Open Platform Communications Undefined Architecture) and PROFINET (Process Field Net).

- OPC UA, developed by OPC Foundation, is an open platform communication based on standard Ethernet for the integration and combination with other standards-based. It considers safety aspects and it ranges from simple process data acquisition to complex monitoring, control, and analysis. OPC UA uses a server-client based communication concept to link the devices to the cloud. $[5,6,7]$

- PROFINET, developed by PROFIBUS \& PROFINET International (PI), is similar to OPC UA as an open communication protocol based on standard Ethernet and considers safety aspects too. The main difference is that PROFINET was designed with a real-time fieldbus to give a deterministic performance for automation applications. With the range of 1 to 10 milliseconds, it can be used to motion control and high-performance requirements. [8, 9,10]

Digital twin is a complex term to describe, Figure 1 tries to illustrate that concept. For those who listen to the term for the first time, it can be described as the virtualization in real-time of the proceeding or task that a machine or a component is doing.

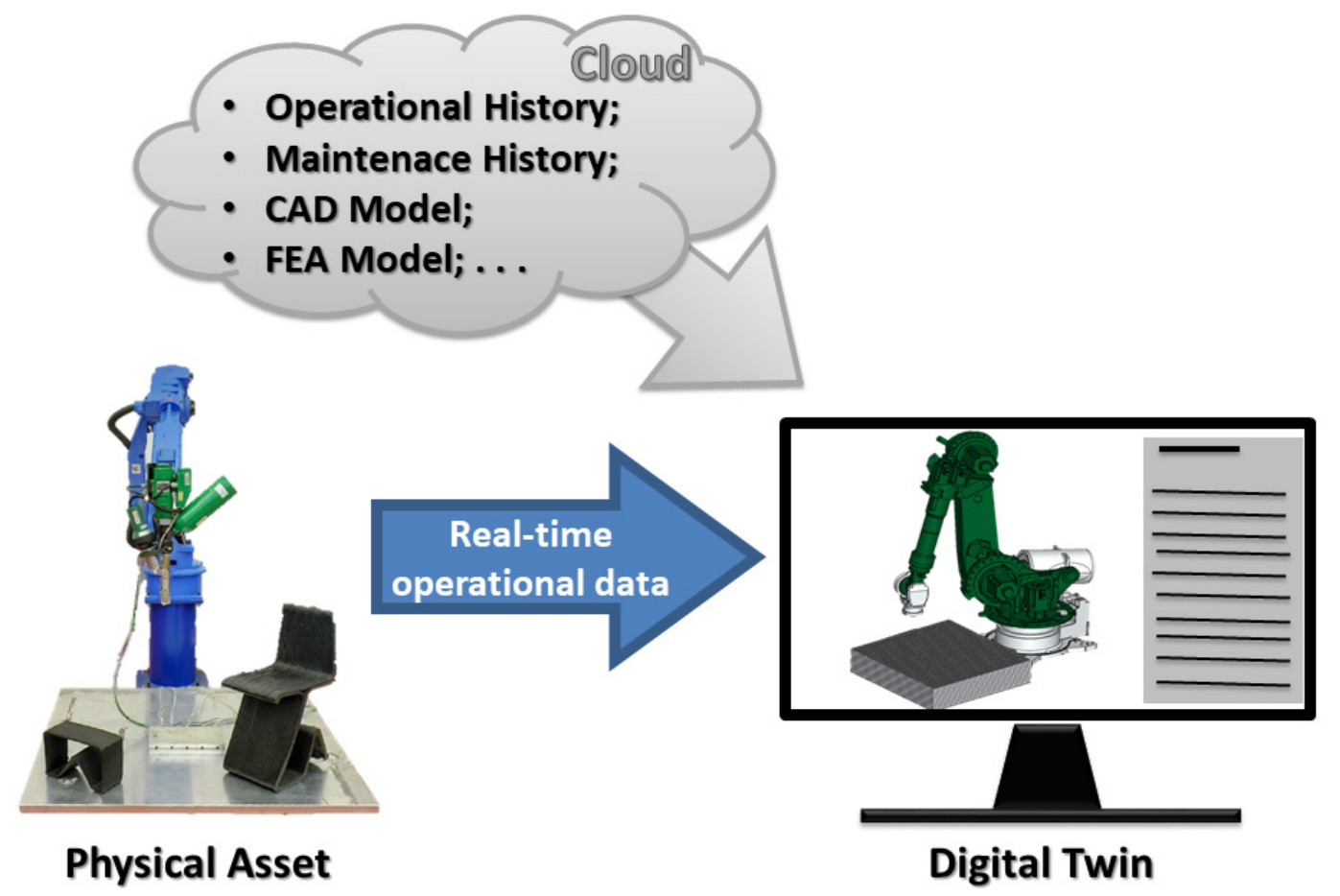

Figure 1 - Digital Twin concept.

Its initial concept was "an integrated multi-physics, multi-scale, probabilistic simulation of a vehicle or system that uses the best available physical models, sensor updates, fleet history, etc., to mirror the life of its flying twin. It is ultra-realistic and may consider one or more important and interdependent vehicle systems", forged by NASA in 2010 [11]. Digital twin collects data to a big data storage or cloud. With that database, digital twin can offer us a choice based on numerical, economic or environmental simulations. Based on those simulations it can also create prediction and warnings. When the choice is chosen it sends the information to the real twin to execute it. [12] According to Elisa Negri et al. [11], the most recent description of digital twin is "A unified system 
model that can coordinate architecture, mechanical, electrical, software, verification, and other discipline specific models across the system lifecycle, federating models in multiple vendor tools and configuration-controlled repositories".

The term of Direct Digital Manufacturing (DDM) appears when the Additive Manufacturing (AM) and IoT ways were crossed. AM is the process of fabricating, usually layer by layer, a product directly from a 3D CAD (Computer-Aided Design) model without the need of process planning [13]. Nowadays DDM can do hybrid manufacturing (Additive and Subtractive), manufacturing products with varied features and sizes, integration with traditional manufacturing systems [14]. AM covers metal, plastic and ceramic materials. AM shows design freedom and not cause material waste (except support structures) as the major advantages when compared to conventional manufacturing. Most of the AM machinery applied in the industry are equipped with three axes, an advantage is using robotic arms to increase the axes [15]. A robotic arm of 6 degrees of freedom can make AM on top of curved surfaces [16]. Hybrid manufacturing, which employs both additive and subtractive technologies, are proliferating because manufacturers recognize that subtractive methods can be used to improve dimensional accuracy, surface finish, and resolution. Most of the hybrid approaches are focused on DDM of metal [17].

In order to every "thing" share its data and have access to data provided by other "things" was developed a Could-Based Manufacturing (CBM) and Could-Based Design and Manufacturing (CBDM). According to Lane Thames and Dirk Schaefer [2], CBM can be described as "a networked manufacturing model that exploits on-demand access to a shared collection of diversified and distributed manufacturing resources to form temporary, reconfigurable cyber-physical production lines which enhance efficiency, reduce product lifecycle costs, and allow for optimal resource allocation in response to variable-demand customer generated tasking". CBDM was developed to store the design and manufacturing resources into a unique data-based cloud. Dirk Schaefer et al. [18] described it as "Cloud-Based Design and Manufacturing refers to a product development model that enables collective open innovation and rapid product development with minimum costs through social networking and crowd-sourcing platforms coupled with shared service pools of design and manufacturing resources and components".

\section{DDM for Big Parts: Digital Twin Approach}

Printing big parts, such as garden benches or urban furniture, are nowadays growing up in rapid manufacturing. Same researches were made to print big parts with green materials as polymeric materials with tire, cork, wood or stone pow loads. [19]

To print big parts, a robotic arm coupled with a $3 \mathrm{D}$ printing tool and a platform with the capacity of adjusting to the final product is suggested. The idea of the adjustable platform is to unused the support material to support the part while printing. The platform can be described as an independent block matrix, where which block was independently controlled. The robotic arm has 6 axes of freedom. With those can be manufactured easier big parts and haven't waste material.

The adjustable platform can adapt each block to the part at each layer, it aims robot moves easier without collapsing with the blocks. Figure 2 shows a garden bench manufactured by a robotic arm and the adjustable platform. 


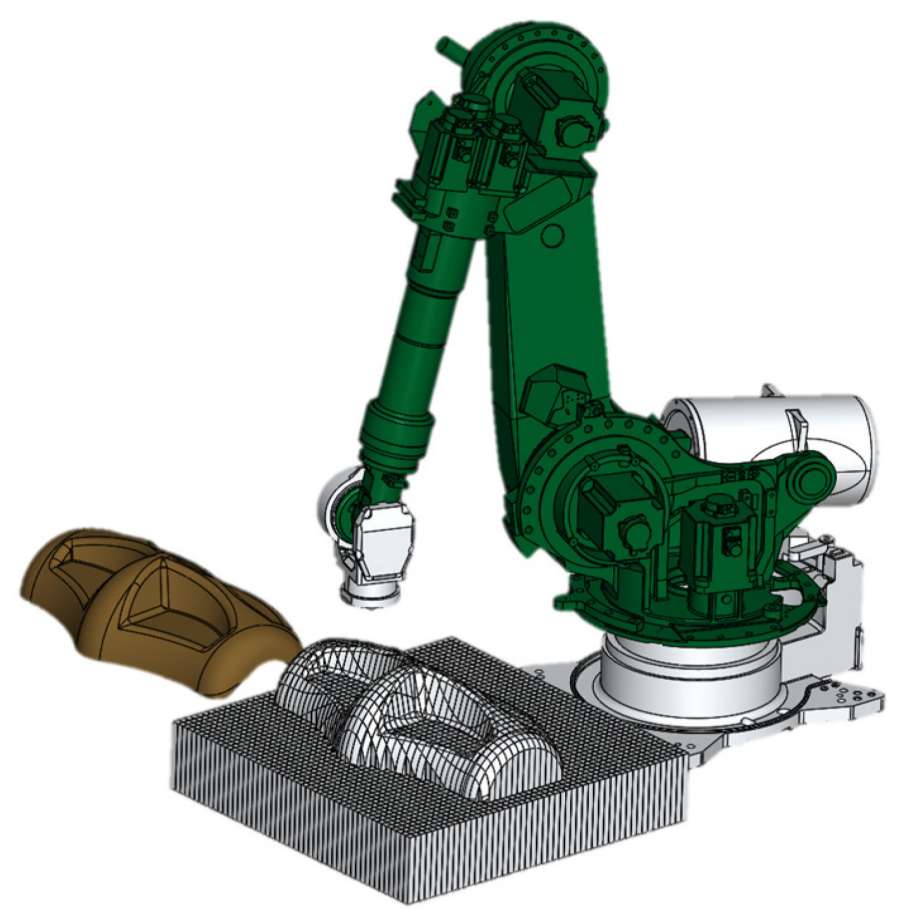

Figure 2 - Big parts printing with robot and adjustable platform.

Approaching a digital twin, the communication between robotic arm, adjustable platform and user needs to be in real-time, making decisions became efficient. This data has to be stored in the cloud for everything access them and create a data-based historical. With that database the digital twin can predict or even warn the user to manufacture the part efficiently, spending less time.

The way to achieve that goal is creating a cloud and connects those via PROFINET protocol. To clarify this idea, Figure 3 represents the implementation measure that is expected.

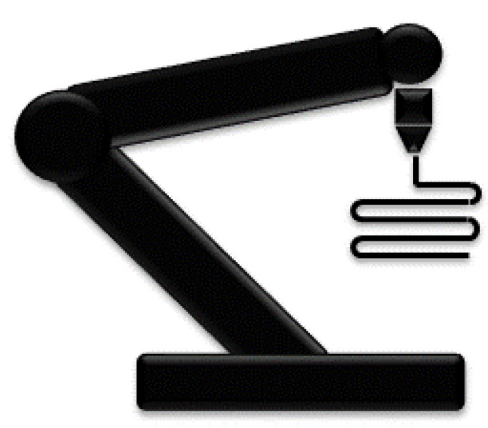

Robot

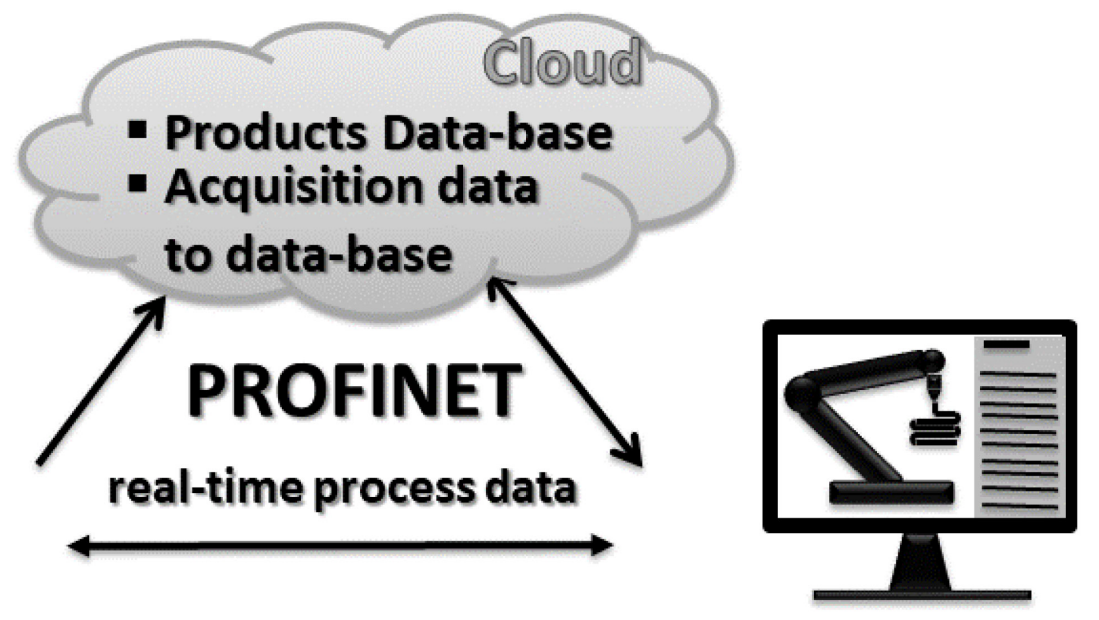

Digital Twin

Figure 3 - Digital Twin and cloud implementation scheme.

Once that step completed, the next objective is creating a cycle from 3D CAD design to real object crossing DDM. The Siemens NX is the software used for mo and conception of the new products. With shared data in a cloud, users are able to develop products and see historic of products. To post-processing the 3D CAD part to the robotic arm, the software Siemens Simatic is used. With the digital twin, the user can communicate and have real-time feedback to control or act if it is necessary. Figure 4 refers to the steps previously mentioned from de modelling to the real object. 


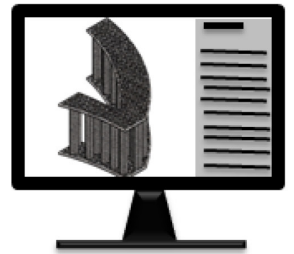

Modelation

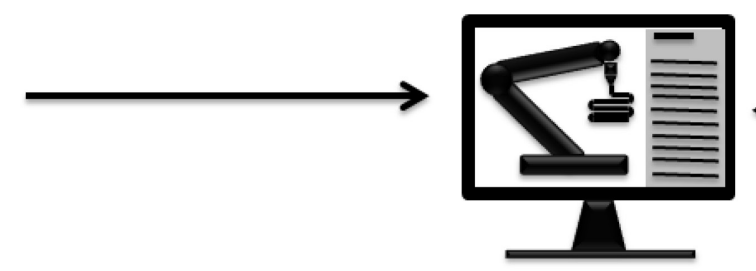

Post-Processor

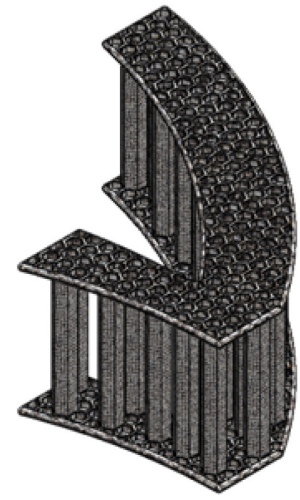

Real object
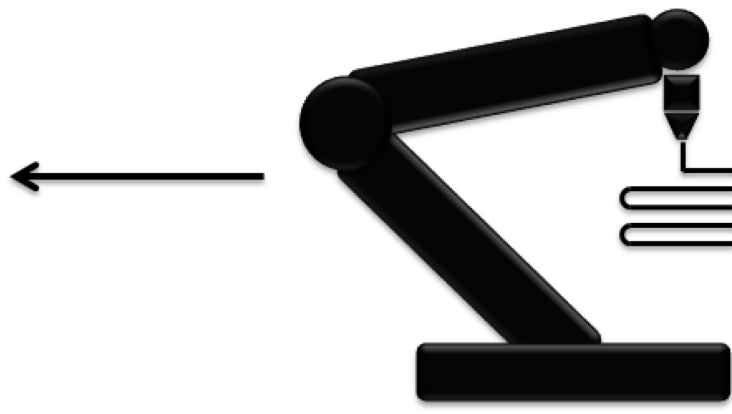

Robot

Figure 4 - Cycle from modelling to real object.

The pretending is to apply what is mentioned in previous chapters to a Yaskawa's robotic arm, the MOTOMAN HP20F with an FS100 controller. Create a cloud and apply the concepts of digital direct manufacturing and digital twin on it. The FS100 controller has a Master/Slaver PROFINET interface [20], in other words, there is no needed to link a PLC between FS100 controller and Ethernet cable to connecting and collecting robot data to the cloud. For now, it is equipped with a 3D printing tool as shown in Figure 5. That tool is an extruder capable of processing pellets instead of wire to manufacture the recycled material. It is controlled directly by the robot so that the process is done automatically [19].

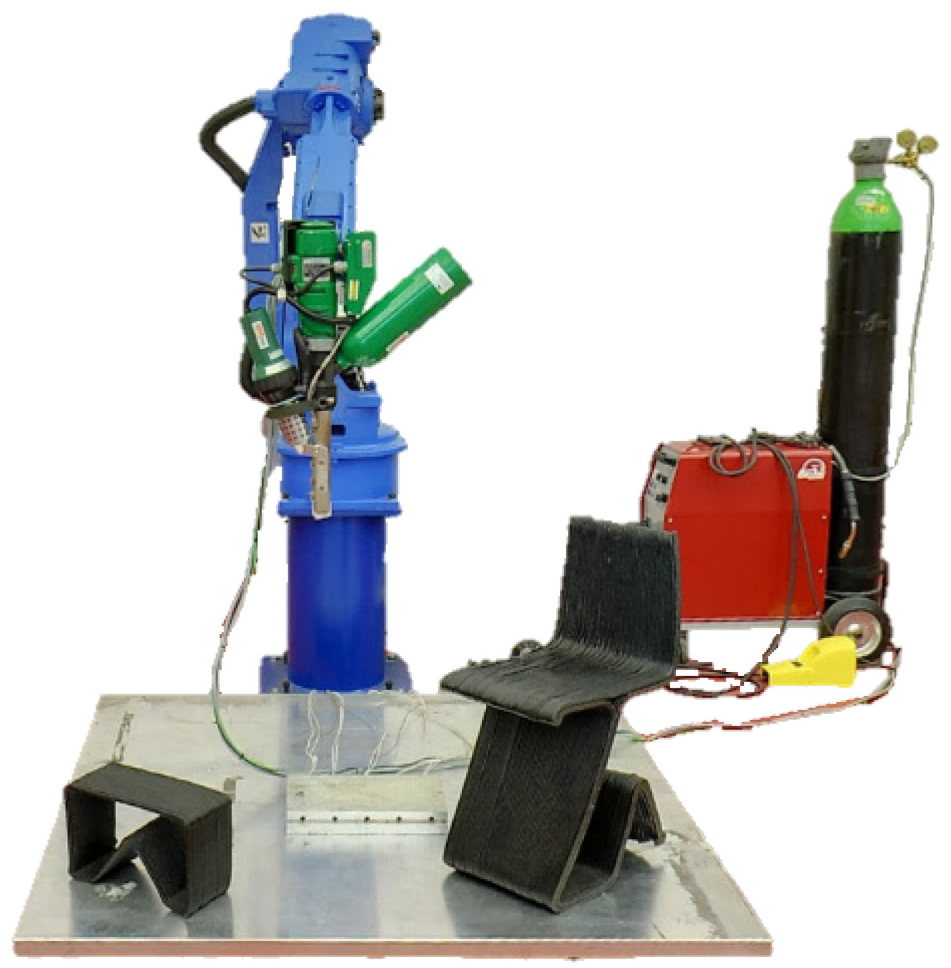

Figure 5 - Image of robotic arm with 3D printing tool. 


\section{Conclusions}

It is expectable that an approach to digital twin can be very useful in a short future. With the existent technology, the industry is able to create his own digital twin with a PROFINET protocol and a cloud or a big storage data. Digital twin is an advantage for human-machine interaction, offline programming with the real environment, improving predictions by simulations made and less time lost in set up. This technology is able to advise user and create and control all process. It is more valuable when applied to create big parts, the process is totally autonomous and the materials can be reused ones as tire, cork, wood or stone pow loads.

As future work, it is intended to extend all those concepts to others industrial machinery such as CNC.

\section{Acknowledgements}

This work is supported by the Fundação para a Ciência e a Tecnologia (FCT) and Centro2020 through the Project references: UID/Multi/04044/2013 and PAMI - ROTEIRO/0328/2013 (N ${ }^{\circ}$ 022158). The authors gratefully acknowledge to OPTIMA and CADFLOW.

\section{References}

[1] V. Roblek, M. Meško, and A. Krapež, “A Complex View of Industry 4.0,” SAGE Open, pp. $1-11,2016$.

[2] L. Thames and D. Schaefer, "Software-defined Cloud Manufacturing for Industry 4.0," Procedia CIRP, vol. 52, pp. 12-17, 2016.

[3] Y. Lu, "Industry 4.0: A survey on technologies, applications and open research issues," $J$. Ind. Inf. Integr., vol. 6, pp. 1-10, 2017.

[4] J. Posada et al., "Visual Computing as a Key Enabling Technology for Industrie 4.0 and Industrial Internet," IEEE Comput. Graph. Appl., pp. 26-40, 2015.

[5] M. Schleipen, S. S. Gilani, T. Bischoff, and J. Pfrommer, "OPC UA \& Industrie 4.0 Enabling Technology with High Diversity and Variability," Procedia CIRP, vol. 57, pp. 315320, 2016.

[6] OPC Foundation, "Unified Architecture." [Online]. Available: https://opcfoundation.org/about/opc-technologies/opc-ua/. [Accessed: 28-Oct-2017].

[7] J. Schmitt, T. Goldschmidt, and P. Vorst, "Cloud-enabled Automation Systems using OPC UA," Atp Ed., vol. 56, pp. 34-41, 2014.

[8] PINorth America (PROFIBUS/PROFINET International), "PROFINET, Industrial Ethernet for advanced manufacturing." [Online]. Available: http://us.profinet.com/technology/profinet/. [Accessed: 28-Oct-2017].

[9] J. Y. Chen, K. C. Tai, and G. C. Chen, "Application of Programmable Logic Controller to Build-up an Intelligent Industry 4.0 Platform," Procedia CIRP, vol. 63, pp. 150-155, 2017.

[10] S. Schriegel, F. Pethig, S. Windmann, and J. Jasperneite, "PROFIanalytics - die Brücke zwischen PROFINET und Cloud-basierter Prozessdatenanalyse," Autom. 2017, Baden-bad., 2017.

[11] E. Negri, L. Fumagalli, and M. Macchi, "A Review of the Roles of Digital Twin in CPSbased Production Systems,” Procedia Manuf., vol. 11, no. June, pp. 939-948, 2017.

[12] J. P. Colin, "Minds + Machines: Meet A Digital Twin," 2016. [Online]. Available: https://www.ge.com/digital/industrial-internet/digital-twin. [Accessed: 18-Oct-2017].

[13] D. Chen, S. Heyer, S. Ibbotson, K. Salonitis, J. G. Steingrímsson, and S. Thiede, "Direct digital manufacturing: Definition, evolution, and sustainability implications," J. Clean. Prod., vol. 107, pp. 615-625, 2015.

[14] A. M. Al-Ahmari, "Direct Digital Manufacturing," J. King Saud Univ. - Eng. Sci., vol. 29, no. 3 , p. $203,2017$. 
[15] M. Rieger, D. D. Störkle, L. Thyssen, and B. Kuhlenkötter, "Robot-Based Additive Manufacturing for Flexible Die-Modelling in Incremental Sheet Forming," Esaform, vol. 40012, 2017.

[16] K. Dröder, J. K. Heyn, R. Gerbers, B. Wonnenberg, and F. Dietrich, "Partial Additive Manufacturing: Experiments and Prospects with Regard to Large Series Production," Procedia CIRP, vol. 55, pp. 122-127, 2016.

[17] S. Ambriz et al., "Material handling and registration for an additive manufacturing-based hybrid system," J. Manuf. Syst., vol. 45, pp. 17-27, 2017.

[18] D. Schaefer, L. Thames, D. Wu, S. Yim, and D. Rosen, "Distributed Collaborative Design and Manufacture in the Cloud-Motivation, Infrastructure, and Education," ASEE 2012 Annu. Conf. Expo., 2012.

[19] J. Domingues, T. Marques, A. Mateus, P. Carreira, and C. Malça, "An Additive Manufacturing Solution to Produce Big Green Parts from Tires and Recycled Plastics," Procedia Manuf., vol. 12, no. December 2016, pp. 242-248, 2017.

[20] Yaskwa Europe, "MOTOMAN FS100 Industrial Robot Controller." [Online]. Available: https://www.yaskawa.eu.com/index.php?eID=dumpFile \&t=f\&f=13337\&token=1a3aae4fef6f d8b66cfb30763545b1e3214c7f91. [Accessed: 09-Nov-2017]. 